\title{
Advanced Operator-Splitting-Based Semi-Implicit Spectral Method to Solve the Binary Phase-Field Crystal Equations with Variable Coefficients
}

\author{
György Tegze* ${ }^{*}$, Gurvinder Bansel ${ }^{a}$, Gyula I. Tóth ${ }^{b}$, \\ Tamás Pusztai ${ }^{b}$, Zhongyun Fan ${ }^{a}$, and László Gránásy ${ }^{a}$ \\ ${ }^{a}$ Brunel Centre for Advanced Solidification Technology, Brunel University, \\ Uxbridge, Middlesex UB8 3PH, United Kingdom \\ ${ }^{b}$ Research Institute for Solid State Physics and Optics, P.O. Box 49, H-1525 \\ Budapest, Hungary
}

\begin{abstract}
We present an efficient method to solve numerically the equations of dissipative dynamics of the binary phase-field crystal model proposed by Elder et al. [Phys. Rev. B 75, 064107 (2007)] characterized by variable coefficients. Using the operator splitting method, the problem has been decomposed into sub-problems that can be solved more efficiently. A combination of non-trivial splitting with spectral semi-implicit solution leads to sets of algebraic equations of diagonal matrix form. Extensive testing of the method has been carried out to find the optimum balance among errors associated with time integration, spatial discretization, and splitting. We show that our method speeds up the computations by orders of magnitude relative to the conventional explicit finite difference scheme, while the costs of the pointwise implicit solution per timestep remains low. Also we show that due to its numerical dissipation, finite differencing can not compete with spectral differencing in terms of accuracy. In addition, we demonstrate that our method can efficiently be parallelized for distributed memory systems, where an excellent scalability with the number of CPUs is observed.
\end{abstract}

Key words: Operator Splitting, Spectral Methods, Phase-Field Crystal PACS: 02.30.Jr, 02.60.Cb, 02.70.Hm, 05.10.-a, 81.10.Aj, 81.16.Dn

\footnotetext{
* Corresponding author. Tel: +44-1895-266411; Fax: +44-1895-269758.

Email address: Gyorgy.Tegze@brunel.ac.uk (György Tegze).
} 


\section{Introduction}

Continuum/field theoretical models have been used widely to address phase transitions in complex systems, including magnetic phase transitions, condensation, phase separation, and crystallization [1-9]. A very promising new field theoretical approach to crystallization of undercooled liquids is the PhaseField Crystal (PFC) model, which addresses freezing on the atomistic/molecular scale $[10,11]$. The PFC method is a close relative of the classical density functional theory (DFT) of crystallization [12]: one may derive it by making a specific approximation for the two-particle direct correlation function of the liquid [11] in the Ramakrishnan-Yussouff expansion of the free energy functional of the crystal relative to the homogeneous liquid [12]. One arrives then to a free energy functional of the Swift-Hohenberg (SH) kind [13]. (In two dimensions, a Brazovsky type free energy functional, valid for triangular lattice emerges [14].) Unlike the original SH model, in the PFC the order parameter is the number density, thus conserved dynamics is assumed to apply $[10,11]$. Remarkably, the PFC description includes automatically the elastic effects and crystal anisotropies, while addressing interfaces, dislocations and other lattice defects on the atomic scale $[10,11,15]$. It also has the advantage over traditional atomistic simulations (such as molecular dynamics), that it works on the diffusive time scale, i.e., processes taking place on about a million times longer times than the ones molecular dynamics simulations are able to handle can be addressed. Thermal fluctuations can be incorporated into the PFC similarly to conventional field theory via adding fluctuation-dissipation noise to the governing equations. Although, due to its atomistic nature, the PFC technique cannot be easily used to model large scale crystalline structures, it has already demonstrated its high potential for modeling dendrites, eutectic structures, polycrystalline solidification, grain boundaries/dislocations, epitaxial growth, crack formation, etc [15]. To address complex solidification morphologies, such as dendritic and eutectic structures, one needs a minimum two-component formulation of the PFC. Very recently, Elder et al. have presented a binary version of the PFC model $[15,16]$. However, the numerical solution of the binary PFC model is rather demanding: even if the direct correlation function of the liquid phase is approximated only to the fourth order in the Fourier space, sixth order stochastic partial differential equations (PDE) need to be solved. More accurate approximations or more complex crystal strucutres yield higher-order PDEs. Numerical solution of such equations requires advanced techniques as demonstrated for the single-component case $[10,11,17]$. It is worth noting that an extra difficulty arises in the binary PFC model: in its full formulation [16], variable coefficients appear in the equations of motion, which cannot be efficiently handled by the numerical methods applied for the single-component case. A different approach is thus required to model binary solidification by the PFC method. Various approaches might be possible at this stage. For example, a combination of the coarse grained formu- 
lation based on the renormalization group technique with adaptive gridding can certainly enhance substantially the simulation domain [18-20]. Unfortunately, a coarse grained version is unavailable yet for the binary PFC. Another possibility is to apply advanced numerical techniques to solve the binary PFC equations.

Recently, the operator splitting techniques are considered as being among the most efficient ones for solving complex PDEs applied in physics [21,22]. A broad range of problems has been addressed by such methods, including the Navier-Stokes equation [23,24], the Hamilton-Jacobi equation [25,26], and advection-diffusion problems $[27,28]$. In these methods, the spatial differential operator is split into a sum of sub-operators that have simpler forms and can be handled easier. Accordingly, the original problem is replaced by a sequence of sub-problems solved numerically. This procedure is efficient though gives rise to some amount of error (splitting error, whos order can be often theoretically estimated [29]). This error is accompanied with the error emerging from the numerical methods used for solving the PDEs of the sub-problems (numerical error). The interaction of these two types of errors determines the total error of the solution. As a result, the method of discretization should be chosen with some care to avoid order reduction and unnecessary loss of accuracy, as investigated recently [30]. Keeping these in mind, the operator splitting methods are considered as promising candidates for solving efficiently the high-order, non-lidear PDEs of the binary PFC model.

In this paper, we are going to apply an advanced operator splitting technique to solve the binary PFC equations efficiently. The rest of the paper is structured as follows. In Section 2, we briefly recall the binary PFC equiations, and describe the numerical thechniques we find propose. In Section 3, we specify the computational resources used, while in Section 4, we present a detailed analysis of the accuracy/efficiency of these methods under conditions leading typically to dendritic growth morphology. Efficiency of the applied numerical methods on parallel computers with distributed memory is also adressed. Finally, a few concluding remarks are made in Section 5.

\section{Binary PFC equations and numerical formulation}

\subsection{Binary PFC theory}

In deriving the binary PFC model, the starting point is the free energy functional of the binary perturbative DFT, where the free energy is Taylor expanded relative to the liquid state (denoted by subscript $L$ ) up to 2 nd order in density difference (up to two-particle correlations) [16]: 


$$
\begin{aligned}
\frac{\mathcal{F}}{k_{B} T}=\int d \vec{r}\left[\rho_{A} \ln \left(\frac{\rho_{A}}{\rho_{L}^{A}}\right)-\Delta \rho_{A}+\rho_{B} \ln \left(\frac{\rho_{B}}{\rho_{L}^{B}}\right)-\Delta \rho_{B}\right] \\
-\frac{1}{2} \int d \vec{r}_{1} d \vec{r}_{2}\left[\Delta \rho_{A}\left(\vec{r}_{1}\right) C_{A A}\left(\vec{r}_{1}, \vec{r}_{2}\right) \Delta \rho_{A}\left(\vec{r}_{2}\right)\right. \\
+\Delta \rho_{B}\left(\vec{r}_{1}\right) C_{B B}\left(\vec{r}_{1}, \vec{r}_{2}\right) \Delta \rho_{B}\left(\vec{r}_{2}\right) \\
\left.+2 \Delta \rho_{A}\left(\vec{r}_{1}\right) C_{A B}\left(\vec{r}_{1}, \vec{r}_{2}\right) \Delta \rho_{B}\left(\vec{r}_{2}\right)\right]
\end{aligned}
$$

where $k_{B}$ is Boltzmann's constant, $\Delta \rho_{A}=\rho_{A}-\rho_{L}^{A}$ and $\Delta \rho_{B}=\rho_{B}-\rho_{L}^{B}$. It is assumed here that all two point correlation functions are isotropic, i.e., $C_{i j}\left(\vec{r}_{1}, \vec{r}_{2}\right)=C_{i j}\left(\left|\vec{r}_{1}-\vec{r}_{2}\right|\right)$. Taylor expanding direct the correlation functions in Fourier space up to 4 th order, one obtains $C_{i j}=\left[C_{i j}^{0}-C_{i j}^{2} \nabla^{2}+C_{i j}^{4} \nabla^{4}\right] \delta\left(\vec{r}_{1}-\vec{r}_{2}\right)$ in real space, where $\nabla$ differentiates with respect to $\vec{r}_{2}$ (see [16]), and $\nabla^{2}$ stands for the Laplacian. The partial direct correlation functions $C_{i j}$ can be related to measured or computed partial structure factors (see e.g. [31]).

Following Elder et al. [16], we introduce the reduced partial number density differences $n_{A}=\left(\rho_{A}-\rho_{L}^{A}\right) / \rho_{L}$ and $n_{B}=\left(\rho_{B}-\rho_{L}^{B}\right) / \rho_{L}$, where $\rho_{L}=\rho_{L}^{A}+\rho_{L}^{B}$. It is also convenient to introduce the new variables $n=n_{A}+n_{B}$ and $\delta N=$ $\left(n_{B}-n_{A}\right)+\frac{\rho_{L}^{B}-\rho_{L}^{A}}{\rho_{L}}$. Then, expanding the free energy around $\delta N=0$ and $n=0$ one obtains

$$
\begin{aligned}
\frac{\mathcal{F}}{\rho_{L} k_{B} T}= & \int d \vec{r}\left(\frac{n}{2}\left[B_{L}+B_{S}\left(2 R^{2} \nabla^{2}+R^{4} \nabla^{4}\right)\right] n\right. \\
& +\frac{t}{3} n^{3}+\frac{v}{4} n^{4}+\gamma(\delta N)+\frac{w}{2}(\delta N)^{2}+\frac{u}{4}(\delta N)^{4} \\
& \left.+\frac{L^{2}}{2}|\vec{\nabla}(\delta N)|^{2}+\cdots\right) .
\end{aligned}
$$

where $B_{S}, t, v, \gamma, w$ and $u$ are constant model parameters, while $B_{L}(\delta N)$ and $R(\delta N)$ represent the variable coefficient part in the kinetic equations as specified below.

Assuming a substitutional diffusion between species $A$ and $B$, i.e., that the same $M$ mobility applies for the two species, the dynamics of the fields $n$ and $(\delta N)$ decouple. Assuming, furthermore, that the mobility coefficient is a constant, the respective equations of motions have the form [16]:

$$
\begin{aligned}
& \frac{\partial n}{\partial t}=M_{e} \nabla^{2} \frac{\delta \mathcal{F}}{\delta n}, \\
& \frac{\partial(\delta N)}{\partial t}=M_{e} \nabla^{2} \frac{\delta \mathcal{F}}{\delta(\delta N)},
\end{aligned}
$$

where $\frac{\delta \mathcal{F}}{\delta \chi}=\frac{\partial I}{\partial \chi}+\sum_{i}(-1)^{i} \nabla^{i} \frac{\partial I}{\partial \nabla^{i} \chi}$ is the first functional derivative of the free 
energy with respect to field $\chi[32]$, and $I$ is the integrand of Eq. (2), $i$ is a positive integer, while the respective effective mobility is $M_{e}=2 M / \rho_{L}{ }^{2}$. Expanding $B_{L}, B_{S}$ and $R$ in terms of $(\delta N)$ with coefficients denoted as $B_{i}^{L}, B_{i}^{S}$ and $R_{i}$, assuming that only coefficient $B_{0}^{L}, B_{2}^{L}, B_{0}^{S}, R_{0}$, and $R_{1}$ differ from zero, and inserting the respective form of $I$ into Eqs. (3) and (4), one arrives at

$$
\begin{aligned}
\frac{\partial n}{\partial t}= & M_{e} \nabla^{2}\left[n\left[B_{0}^{L}+B_{2}^{L}(\delta N)^{2}\right]+t n^{2}+v n^{3}\right. \\
& +\frac{B_{0}^{S}}{2}\left\{2\left[R_{0}+R_{1}(\delta N)\right]^{2} \nabla^{2}+\left[R_{0}+R_{1}(\delta N)\right]^{4} \nabla^{4}\right\} n \\
& \left.+\frac{B_{0}^{S}}{2}\left\{2 \nabla^{2}\left(n\left[R_{0}+R_{1}(\delta N)\right]^{2}\right)+\nabla^{4}\left(n\left[R_{0}+R_{1}(\delta N)\right]^{4}\right)\right\}\right] \\
\frac{\partial(\delta N)}{\partial t}= & M_{e} \nabla^{2}\left[B_{2}^{L}(\delta N) n^{2}\right. \\
& +2 B_{0}^{S} n\left\{\left[R_{0}+R_{1}(\delta N)\right] R_{1} \nabla^{2}+\left[R_{0}+R_{1}(\delta N)\right]^{3} R_{1} \nabla^{4}\right\} n \\
& \left.+\gamma+w(\delta N)+u(\delta N)^{3}-L^{2} \nabla^{2}(\delta N)\right] .
\end{aligned}
$$

Eqs. (5) and (6) will be solved numerically after adding a conservative noise (a random flux) to them that represents the thermal fluctuations.

Finally, we briefly outline the physical meaning of the model parameters. The driving force of crystallization can be enhanced by either lowering $\Delta B=$ $B_{0}^{L}-B_{0}^{S}$ (lowering the temperature), or increasing the initial number density $\bar{n}$ (incresing the pressure), or by changing the initial composition of the liquid phase $\overline{\delta N}$. The magnitude of parameter $t$ is determined by the interplay of the appropriate Taylor coefficient of the ideal gas term in the free energy with the 0 -th order contribution from the three-particle correlation, while the magnitude of $v$ follows from the Taylor coefficent for the logarithmic term in the ideal gas contribution. The interatomic distance may be tuned via parameters $R_{0}$ and $R_{1}$, of which the latter determines the composition dependence of the interatomic spacing. The tendency towards liquid/solid phase separation can be tuned by changing $w$, while the lenght scale of phase separation is determined by the interplay of $L, w$, and $u$.

\subsection{The numerical scheme}

In order to simplify the complex equations of motion [Eqs. (5) and (6)], we apply differential splitting prior to discretization. 


\subsubsection{Operator splitting}

We decompose the spatial differential operator in the sum of two operators as follows:

$$
\begin{gathered}
\frac{\partial n}{\partial t}=\left(\mathbf{A}_{\mathbf{1}}+\mathbf{A}_{\mathbf{2}}\right) n \\
\frac{\partial(\delta N)}{\partial t}=\left(\mathbf{B}_{\mathbf{1}}+\mathbf{B}_{\mathbf{2}}\right)(\delta N) .
\end{gathered}
$$

while sub-operators $\mathbf{A}_{\mathbf{1}}, \mathbf{A}_{\mathbf{2}}, \mathbf{B}_{\mathbf{1}}$ and $\mathbf{B}_{\mathbf{2}}$ have the form:

$$
\begin{aligned}
\mathbf{A}_{1} n & =\nabla^{2}\left\{\left[M_{e}\left\{B_{0}^{L}+B_{2}^{L}(\delta N)^{2}\right\}-C_{1}\right] n\right\} \\
& +\nabla^{2}\left\{\left[M_{e} B_{0}^{S}\left\{R_{0}+R_{1}(\delta N)\right\}^{2}-C_{2} / 2\right] \nabla^{2} n\right\} \\
& +\nabla^{2}\left\{\left[M_{e} \frac{B_{0}^{S}}{2}\left\{R_{0}+R_{1}(\delta N)\right\}^{4}-C_{3} / 2\right] \nabla^{4} n\right\} \\
& +\nabla^{4}\left\{\left[M_{e} B_{0}^{S}\left\{R_{0}+R_{1}(\delta N)\right\}^{2}-C_{2} / 2\right] n\right\} \\
& +\nabla^{6}\left\{\left[M_{e} \frac{B_{0}^{S}}{2}\left\{R_{0}+R_{1}(\delta N)\right\}^{4}-C_{3} / 2\right] n\right\} \\
& +\nabla^{2}\left\{M_{e}\left[t n^{2}+v n^{3}\right]\right\}, \\
\mathbf{A}_{2} n & =C_{1} \nabla^{2} n+C_{2} \nabla^{4} n+C_{3} \nabla^{6} n, \\
\mathbf{B}_{1}(\delta N) & =M_{e} \nabla^{2}\left[B_{2}^{L}(\delta N) n^{2}\right. \\
& +2 B_{0}^{S} n\left\{\left[R_{0}+R_{1}(\delta N)\right] R_{1} \nabla^{2}+\left[R_{0}+R_{1}(\delta N)\right]^{3} R_{1} \nabla^{4}\right\} n \\
& \left.+\gamma+u(\delta N)^{3}\right], \\
\mathbf{B}_{2}(\delta N) & =M_{e}\left[w \nabla^{2}(\delta N)-L^{2} \nabla^{4}(\delta N)\right],
\end{aligned}
$$

where $C_{1}, C_{2}$ and $C_{3}$ are constants to be defined later.

The motivation for this specific choice of split operators is that, with appropriate spatial discretization schemes, it leads to algebraic equations of a diagonal matrix form, which can be solved very easily and efficiently in a pointwise manner. To achieve this, we collect the problematic (non-linear/variable coefficient) terms into sub-operators $\mathbf{A}_{\mathbf{1}}$ and $\mathbf{B}_{\mathbf{1}}$, which can be then solved by explicit FD or spectral schemes (we will present results for the fully spectral approach), while the rest (sub-problems corresponding to sub-operators $\mathbf{A}_{\mathbf{2}}$ and $\mathbf{B}_{\mathbf{2}}$ ) will be handled by implicit spectral methods. As it will be shown, this specific combination of operator splitting with spectral schemes enables 
us to use time steps that are substantially larger than those allowed by the explicit fomulation, and also the accuray of the solution is significantly improved relative to the finite difference spatial discretization.

\subsubsection{Splitting procedure and discretization}

There exist several splitting procedures [21,22,29,30,33-35]. In solving the problem, in this work, we rely on the simplest sequential splitting procedure $[29,30]$. Combining sequential splitting with the explicit time integration for $\mathbf{A}_{1}$ and $\mathbf{B}_{1}$ and implicit time integration for $\mathbf{A}_{2}$ and $\mathbf{B}_{2}$ yielding the following equations:

$$
\begin{gathered}
n^{*}=n^{t}+\Delta t \mathbf{A}_{\mathbf{1}} n^{t} \\
n^{t+\Delta t}=n^{*}+\Delta t \mathbf{A}_{\mathbf{2}} n^{t+\Delta t} \\
(\delta N)^{*}=(\delta N)^{t}+\Delta t \mathbf{B}_{\mathbf{1}}(\delta N)^{t}, \\
(\delta N)^{t+\Delta t}=(\delta N)^{*}+\Delta t \mathbf{B}_{\mathbf{2}}(\delta N)^{t+\Delta t},
\end{gathered}
$$

where $n^{*}$ and $(\delta N)^{*}$ are the solutions of the split sub-problems corresponding to sub-operators $\mathbf{A}_{1}$ and $\mathbf{B}_{1}$.

Note that Eqs. (13) and (15) contain nonlinear terms and terms of variable coefficient. In their spatial discretization we have repeatedly applied fast Fourier transformations (FFTs), differentiation in the spectral space, and inverse fast Fourier transformations (IFFTs). This approach leads to a numerical formulation that is free of numerical dissipation, and to a solution that is far more accurate than the solution relying on the finite difference technique. In 2D Fourier space, the discretized Laplacian coresponds to a multiplication by $-4 \pi^{2}\left(k_{x}^{2}+k_{y}^{2}\right)$, where $k_{x}$ and $k_{y}$ are the discrete wavenumbers. Note, furthermore, that the explicit time integration applied for Eqs. (13) and (15) yields algebraic equations that can be directly written into a diagonal matrix form and solved thus pointwise via simple back-substitution.

In the case of sub-operators containing only constant coefficient terms $\left(\mathbf{A}_{\mathbf{2}}\right.$ and $\mathbf{B}_{\mathbf{2}}$ ), the $2 \mathrm{D}$ spatial discretization has been made using a spectral differencing scheme:

$$
\begin{aligned}
\tilde{n}_{\left(k_{x}, k_{y}\right)}^{t+\Delta t} & =\tilde{n}_{\left(k_{x}, k_{y}\right)}^{*}\left\{1-\Delta t\left(C_{1} 2^{2} \pi^{2}\left(-k_{x}^{2}-k_{y}^{2}\right)\right.\right. \\
& +C_{2} 2^{4} \pi^{4}\left(k_{x}^{4}+2 k_{x}^{2} k_{y}^{2}+k_{y}^{4}\right)
\end{aligned}
$$




$$
\begin{aligned}
& \left.+C_{3} 2^{6} \pi^{6}\left(-k_{x}^{6}-3 k_{x}^{4} k_{y}^{2}-3 k_{x}^{2} k_{y}^{4}-k_{y}^{6}\right)\right\}^{-1}, \\
& \widetilde{(\delta N)}_{\left(k_{x}, k_{y}\right)}^{t+\Delta t}=\widetilde{(\delta N)}_{\left(k_{x}, k_{y}\right)}^{*}\left\{1+\Delta t M_{e} w 2^{2} \pi^{2}\left(k_{x}^{2}+k_{y}^{2}\right)\right. \\
& \left.+\Delta t M_{e} L^{2} 2^{4} \pi^{4}\left(k_{x}^{4}+2 k_{x}^{2} k_{y}^{2}+k_{y}^{4}\right)\right\}^{-1},
\end{aligned}
$$

where $\tilde{n}_{\left(k_{x}, k_{y}\right)}$ and $\widetilde{(\delta N)}\left(k_{x}, k_{y}\right)$ stand for the discrete Fourier transforms of $n(\mathbf{r})$ and $(\delta N(\mathbf{r}))$ at the discrete wave vector $\mathbf{k}=\left(k_{x}, k_{y}\right)$.

We emphasize that in a fully explicit treatment the stepsize one may use for time integration is seriously limited. In contrast, in the present mixed explicitimplicit formulation, we have some freedom to tune the stability criteria to our favour, via a proper choice of the constants $C_{1}, C_{2}$ and $C_{3}$, while retaining the diagonal matrix form of the the algebraic equations. While the stability of time stepping with the individual sub-operators is a necessary condition, due to a possible interaction of errors this does not neccessarily guarantee the overall stability of the scheme [30]. We find though in practice that for the splitting of the PFC equations described above, it is sufficient to ensure the stability of stepping with the operators individually. Next, we are going to examine the stability of the steppings with the indiviual operators, and give suggestions for the proper choice of the splitting constants $C_{1}, C_{2}$ and $C_{3}$.

As a result of sequential operator splitting, and of the spectral implicit treatment of the fourth order term in Eq. (6), the maximum stable time step is proportional to $(\Delta x)^{2}$ that compares favorably to the $\Delta t \propto(\Delta x)^{4}$ dependence of a fully explicit scheme.

The stability of time stepping for Eq. (5) is a more complicated issue, and we also need to address the consistency of the explicit-implicit stepping.

After some manipulation of our equations it can be shown that we have added the terms $\Delta t M_{e}\left[C_{1}\left(\nabla^{2} n^{t+1}-\nabla^{2} n^{t}\right)+C_{2}\left(\nabla^{4} n^{t+1}-\nabla^{4} n^{t}\right)+C_{3}\left(\nabla^{6} n^{t+1}-\nabla^{6} n^{t}\right)\right]$ to the fully explicit discretization of the equation. For $\Delta t \rightarrow 0$ this extra term converges to zero, so the consistency of the scheme is indeed ensured.

Next we specify the coefficients $C_{1}, C_{2}$ and $C_{3}$ of the sub-operator $\mathbf{A}_{1}$ [Eq. (9)] in a way that ensures the stability of time-steppings.

$$
\begin{aligned}
& C_{1}=\left|M_{e}\left\{B_{0}^{L}+B_{2}^{L}(\delta N)^{2}\right\}\right|_{\text {max }} \\
& C_{2}=\left|2 M_{e} B_{0}^{S}\left\{R_{0}+R_{1}(\delta N)\right\}^{2}\right|_{\max } \\
& C_{3}=\left|M_{e} B_{0}^{S}\left\{R_{0}+R_{1}(\delta N)\right\}^{4}\right|_{\max }
\end{aligned}
$$


For every $\Delta t$ timestep and $\Delta x$ mesh spacing one may chose these constants so that the explicit terms with linear variable coefficients are stable. Proper choices of these constants modify the stability criteria. In fact, they push the maxima of the variable coefficients of the second and the sixth order terms into the range, where the differencing terms are stable at a given $\Delta t$ and $\Delta x$. Note that all the variable coefficients are positive in practice, therefore, splitting of the fourth order term is not required to ensure the stability of the explicit stepping. The stability of the explicit stepping can be assured by setting $C_{1}$ and $C_{3}$ as in Eq. (19) and Eq. (21).

However, stability has to be ensured for the implicit stepping too. The respective stability criterion depends on the wavenumber $k$ and can be formulated as $\Delta t M_{e}\left[C_{1} 2^{2} \pi^{2}\left(k_{x}^{2}+k_{y}^{2}\right)-C_{2} 2^{4} \pi^{4}\left(k_{x}^{2}+k_{y}^{2}\right)^{2}+C_{3} 2^{6} \pi^{6}\left(k_{x}^{2}+k_{y}^{2}\right)^{3}\right]>-1$, a condition that restricts the value of $C_{2}$.

Regarding the splitting error, we have found that mixing of the explicit and implicit terms within the numerical scheme should be avoided as much as possible. Here, we may utilize the fact that the realative variation of the coefficients of the equations is small, since the change of $R=R_{0}+R_{1}(\delta N)$ that represents the composition dependence of the interatomic spacing, is small itself (a few percent typically). Then, if $C_{2}$ is chosen as specified by Eq. (20), the variable coefficient terms are treated dominantly in an implicit manner, while the explicit part represents just a small correction. For our particular case the value of $C_{2}$ given by Eq. (20) statisfies also the stability criterion for implicit stepping.

When applying the above scheme to Eq. (5), the overall stability is limited by the nonlinear terms, a restriction, which is usually much less severe than the explicit time integration of the higher order derivatives present in the equation. In practice, the timestep is limited by the accuracy of time integration.

Due to the highly non-linear nature of the equations of motion, nonlinear instability of the numerical solution might appear and indeed occurs under various choices of the model parameters. To handle this, we have used a spherical spectral filter [36] on the non-linear terms in Eq. (6). The filtering has been done by cancelling the frequency components that statisfy the $k_{x}^{2}+k_{y}^{2}>K^{2}$ inequality, where $K$ is a constant defined empirically so as to avoid a catastrophic accumulation of errors at high frequencies.

In Section 4, we are going to compare our method [which we name henceforth a semi-implicit spectral (SIS) approach] to the explicit FD discretization (EFD) in terms of accuracy, stability and the overall computational efficiency in a massively parallel environment. For the FD discretization of the Laplacian, we have used the compact formula below [11]: 


$$
\begin{aligned}
\nabla^{2} f_{i, j} & =\left[\left(f_{i+1, j}+f_{i-1, j}+f_{i, j+1}+f_{i, j-1}\right) / 2,\right. \\
& \left.+\left(f_{i+1, j+1}+f_{i-1, j+1}+f_{i+1, j-1}+f_{i-1, j-1}\right) / 4-3 f_{i, j}\right] /(\Delta x)^{2} .
\end{aligned}
$$

\subsection{Treatment of noise}

We have added a conservative Gaussian noise colored in space to the governing equations in the Fourier space [37], whos amplitude scales with the time step and the cut-off wavelength [38]. In order to avoid the appearance of unphysically small wavelengths, we have applied a cut-off in the Fourier space that removed the wavelengths shorter than the interatomic distance.

\section{Numerical implementation/computing}

Parallel C codes relying on the MPI protocol have been developed to solve the governing equations numerically on an $N \times N$ rectangular grid, using both the SIS and the EFD schemes. To optimize the performance, we have developed a parallel FFT code based on the FFTW3 library [39]. We have prescribed periodic boundary conditions.

The initial conditions for simulations of binary solidification have been created as follows. First, the simulation window has been filled uniformly with appropriate total number density $n=\bar{n}$ and number density difference values $(\delta N)=\overline{\delta N}$ specified in Table 1 , representing the initial undecooled liquid. Next, in the case of dendritic structures, we have placed a small crystalline cluster of 13 density peaks to the center on a hexagonal lattice (central atom + first and second neighbor shells) of suitable interactomic spacing, that has acted as a crystal seed. In the case of eutectic solidification, we have used two seeds of different compositions $[(\delta N)=-0.3$ and 0.3 , respectively] containing 6 density peaks each (central atom + first neighbor shell), placed in contact with each other at the center of the simulation window.

The numerical investigations presented in this paper have been performed using two PC clusters: (i) One at the Research Institute for Solid State Physics and Optics (RISSPO), Budapest, Hungary, that consists of 24 PCs, equipped with two $2.33 \mathrm{GHz}$ Intel processors of $4 \mathrm{CPU}$ cores (192 CPU cores in all on the 24 nodes), 8 GB memory/node, and is equipped with $10 \mathrm{Gbit} / \mathrm{s}$ (InfiniBand) communication, and (ii) another hosted by the Brunel Centre for Advanced Solidification Technology (BCAST), Brunel University, West London, UK, which consists of 20 similar nodes (160 CPU cores), however, with $1 \mathrm{Gbit} / \mathrm{s}$ (standard GigaBit Ethernet) communication in between. 


\section{Results and discussion}

The proposed SIS method will be compared with EFD discretization under conditions that lead to dendritic solidification, a case that clearly demonstrates the potential of the PFC method. Dendritic and eutectic structures grown using the SIS approach are shown in Fig. 1. The respective choices of parameters are given in Table 1 . Here $\zeta_{0}$ is the amplitude of the conservative Gaussian colored noise added to the equations of motion, while a cut-off for wavelengths smaller than $\lambda=7 \Delta x$ has been made in Fourier space. The same spatial steps and 32 times larger time steps have been chosen than in [16], where $\Delta x_{0}=1.1$ and $\Delta t_{0}=0.05$. (We note here that at the time and spatial steps used in [16] the EFD computations are just stable.) The computations for dendritic structures [Figs. 1(a) and 1(c)] have been performed on $20 \times 4 \times 2$ $=160 \mathrm{CPU}$ cores of the RISSPO cluster equipped with $10 \mathrm{Gbit} / \mathrm{s}$ communication, and took about 4 days each. The eutectic computation [Fig. 1(c)] has been performed on $40 \mathrm{CPU}$ cores with $1 \mathrm{Gbit} / \mathrm{s}$ communication, and took $\sim$ 10 days.

Note that in Figs. 1(a) and 1(c) the primary dendrite arms show an almost perfect six-fold symmetry: the lengths of the six dendite arms differ by only $\sim$ $0.1 \%$. Using the SIS scheme, the anisotropy induced by the discretization on a rectangular lattice is negligible, which enables us to predict the morphology of dendritic/eutectic self-organizanized structures accurately.

\subsection{Analysis of the numerical solution}

We are unaware of any non-trivial analitical solution for the binary PFC equations that could be used as a reference in computing the numerical error. Because of the practical difficulties [the EFD computations are severely restricted by the fact that the stability criterion for explicit discretization requires $\left.\Delta t \propto(\Delta x)^{6}\right]$, one can neither obtain a sufficiently accurate EFD solution that could serve as reference. Therefore, first we perform an empirical convergence test (see e.g., [41]) to investigate whether there exists a limiting solution, to which the SIS solutions converge for decreasing $\Delta x$ and $\Delta t$. Finding such a behavior, we use the limiting solution as reference in defining the numerical error. We also attempt to clarify whether the SIS and EFD methods converge to each other for decreasing spatial and time steps in the $\Delta x$ and $\Delta t$ domain available for numerical simulations.

Along these lines, first, we investigate the effect of spatial and time resolution on the numerical solutions obtained by the SIS method. Since we are interested in a quantitative comparison between computations made with different time 

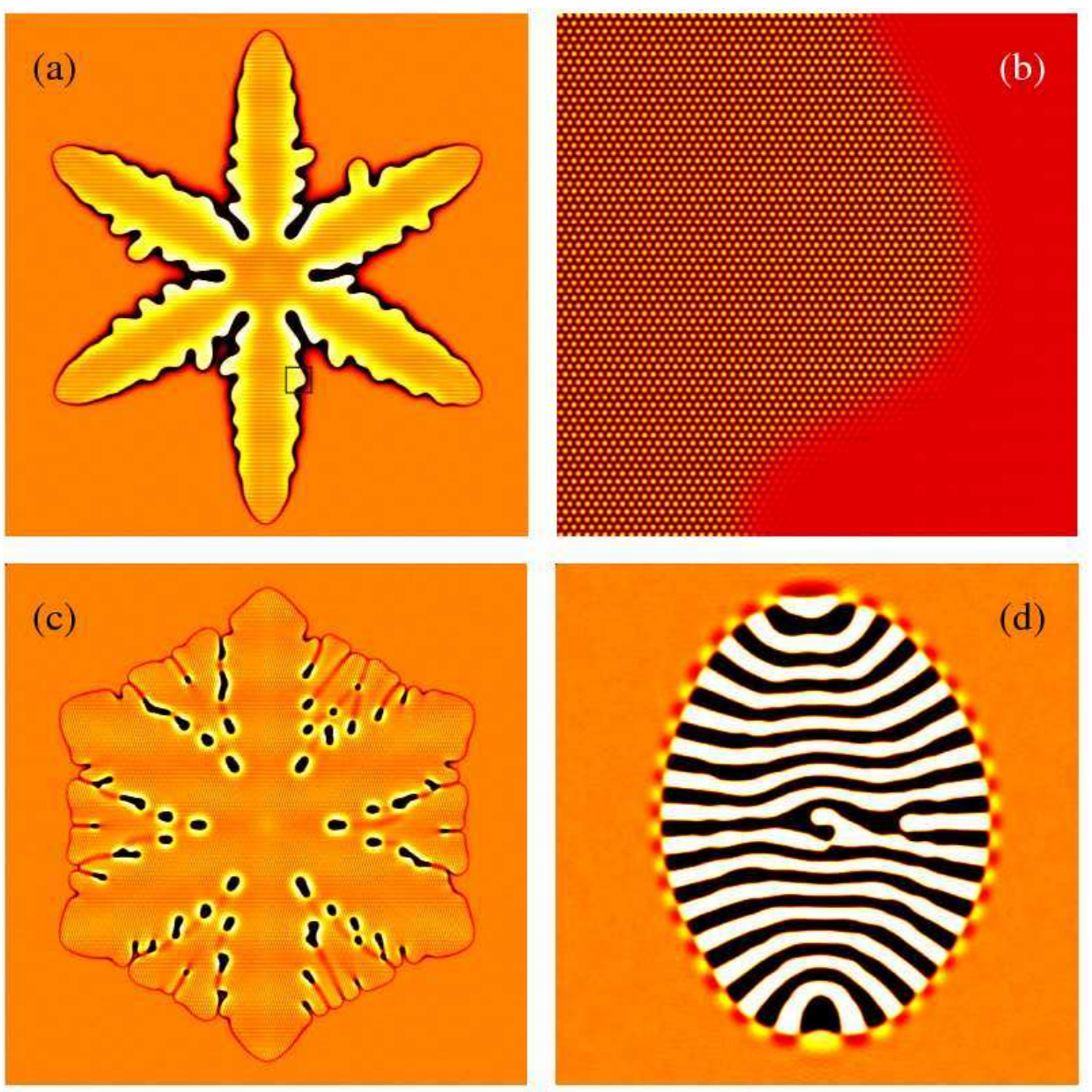

Fig. 1. Illustrative phase-field crystal simulations for solidification in binary alloys. (a) Number density difference $(\delta N)$ map of a solutal dendrite. (b) Total number density $n$ map of the small square area of black border on the right hand side of the downward pointing arm of the dendrite shown in panel (a). (c) A more compact dendritic structure grown at a higher driving force achieved by increasing the initial liquid density relative to panel (a). (d) Eutectic structure obtained by reducing the initial number density $\bar{n}$ and number density difference $\overline{\delta N}$ relative to panel (a). The snapshots shown have been taken after (a),(b) 92,160, (c) 55,000, and (d) 498,000 time steps. The model parameters used in these simulations are listed in Table 1.

and spatial steps and wish to avoid differences of stochastic origin, in all the following simulations, we have switched off the noise that represents the thermal fluctuations. To explore the resolution dependence, we have performed a series of simulations with the proposed numerical scheme on a relatively small physical domain of dimensionless area $281.6 \times 281.6$ (consisting of about 6,600 atoms). This size is a result of a compromise: It is big enough to have bulk crystalline properties at the center of the growing crystallite at dimensionless time $t=768$, and is small enough to allow a few refinement steps in the spatial resolution even for the EFD method: For our study, we have chosen the spatial steps $\Delta x=(1 / 4,1 / 3,1 / 2,2 / 3,3 / 4$, and 1$) \times \Delta x_{0}$. For each of 
Table 1

Parameters used in computing Fig. 1

\begin{tabular}{rrrr}
\hline \hline & $(a),(b)$ & $(c)$ & $(d)$ \\
\hline $\bar{n}$ & 0.0092 & 0.0096 & 0.0 \\
$\overline{\delta N}$ & 0.0904 & 0.0904 & $10^{-6}$ \\
$B_{0}^{L}$ & 1.04 & 1.04 & 1.0248 \\
$B_{2}^{L}$ & -1.8 & -1.8 & -1.8 \\
$B_{0}^{S}$ & 1.0 & 1.0 & 1.0 \\
$R_{0}$ & 1.0 & 1.0 & 1.0 \\
$R_{1}$ & 0.25 & 0.25 & 0.25 \\
$t$ & -0.6 & -0.6 & -0.6 \\
$v$ & 1.0 & 1.0 & 1.0 \\
$\gamma$ & 0.0 & 0.0 & 0.0 \\
$w$ & 0.088 & 0.088 & 0.0 \\
$u$ & 4.0 & 4.0 & 4.0 \\
$L$ & 1.2 & 1.2 & 1.2 \\
$\Delta x / \Delta x_{0}$ & 1.0 & 1.0 & 1.0 \\
$\Delta t / \Delta t_{0}$ & 32.0 & 32.0 & 32.0 \\
$\zeta_{0}$ & $10^{-6}$ & $10^{-6}$ & $10^{-5}$ \\
$N$ & 8,192 & 8,192 & 2,048 \\
\hline & & &
\end{tabular}

these spatial steps SIS simulations have been performed with the time steps $\Delta t=2^{j} \times \Delta t_{0}$, where $j=0,1,2, \ldots, 8$. For comparison, we have made explicit FD computations with the same spatial steps, however, with the largest time steps, allowed by the numerical stability of the explicit scheme. Unfortunately, due to the limited computational capacity available and the very small time steps occuring due to the high order differential operators, we were unable to perform the EFD simulation for the finest mesh spacing.

This analysis of the numerical solution has been performed with the model parameters used in computing the dendritic struture shown in Fig. 1(a), however, without adding noise to the equations of motion $\left(\zeta_{0}=0\right)$.

Characterization of the solution: We find that crystallization is fairly isotropic on this size scale [Fig. 2(a)]. Accordingly, we use the diameter of the crystal $d$ at dimensionless time $t=768$ as a measure of the average growth rate, a quantity that not only characterizes the solution on a mesoscopic scale, but 

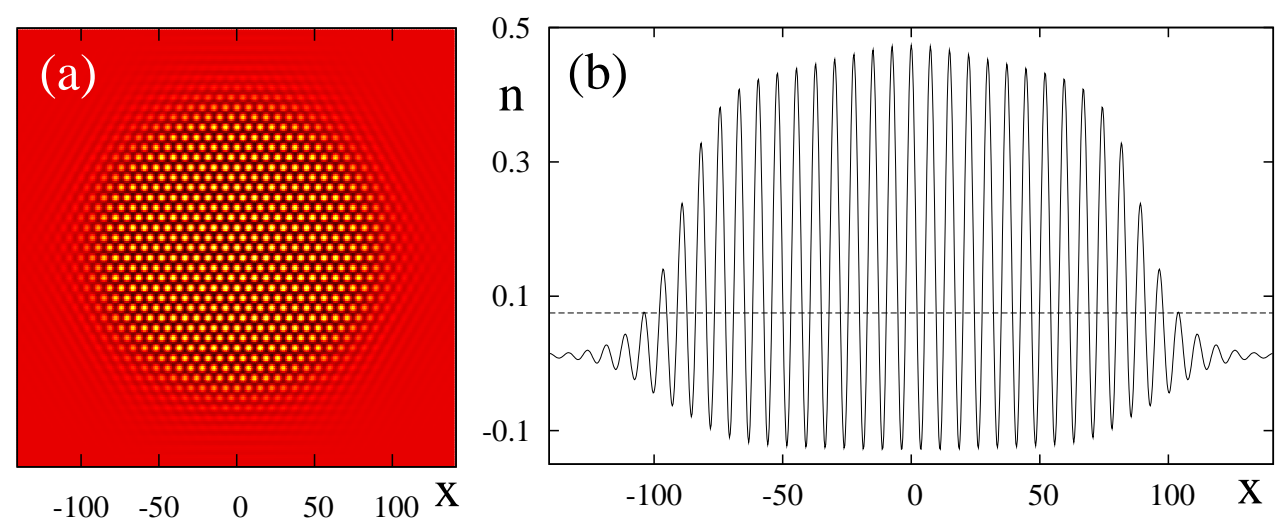

Fig. 2. High resolution SIS solution used as reference: (a) Snapshot of the total number density at time $t=768$ for a simulation performed on a $1024 \times 1024$ grid with $\Delta x=\Delta x_{0} / 4$ and $\Delta t=\Delta t_{0}$; (b) the respective total number density $n$ distribution along the horizontal centerline. The level $n=0.075$ chosen to define the diameter of the crystalline particle is marked by the dashed horizontal line.

also reflects the time evolution of the solution. Due to the atomistic nature of the crystal structure and the gradual transition between the crystal and the homogeneous liquid, the diameter of the crystalline particles has to be defined with some care. Various methods can be devised to deduce the linear size of a crystalline particle. In this work, the diameter of the roughly circular particles has been calculated by connecting the maxima of the neighboring total number density peaks along the horizontal centerline of the particle (lying on a crystal plane) by straight lines, and then taking the intersection of the resulting "peak envelope" with the arbitrary threshold of $n=0.075$ chosen as the limit between the solid and liquid phases. [See Fig. 2(b). Note also the diffuse solid-liquid interface and the close similarity to the number density profiles obtained from molecular dynamics simulations [42-45].] Since the uncertainty of the peak positions is $\sim 2 \Delta x$ on both ends of the diameter, which in turn is $\sim 175 \Delta x_{0}$, the relative error of the diameter is $\pm 2 \Delta x /\left(175 \Delta x_{0}\right)$ that varies in the $\pm 0.3 \%$ to $\pm 1.1 \%$ range. Besides the particle diameter, we use the interatomic distance a (distance between the neighboring number density peaks) to characterize the periodic nature of the solution in the crystal, probing it on the atomic scale. It has been determined by measuring the total length of 10 density waves in a crystal plane $\left(10 a \approx 68 \Delta x_{0}\right)$. Here the reading error is about $2 \Delta x$ for the whole length, $10 a$. So the relative error is $\sim \pm \Delta x /\left(68 \Delta x_{0}\right)$, which ranges between $\pm 0.4 \%$ and $\pm 1.5 \%$. We note that the investigated quantities $(a$ and $d$ ) have physical significance: the interatomic distance reflects the effective atomic interaction the approximate direct correlation function of the PFC model realizes, while the average growth rate monitors the kinetics of the phase transition.

Results: The time and spatial step dependencies of these quantities are presented in a normalized form in Fig. 3. Remarkable features of the SIS results 

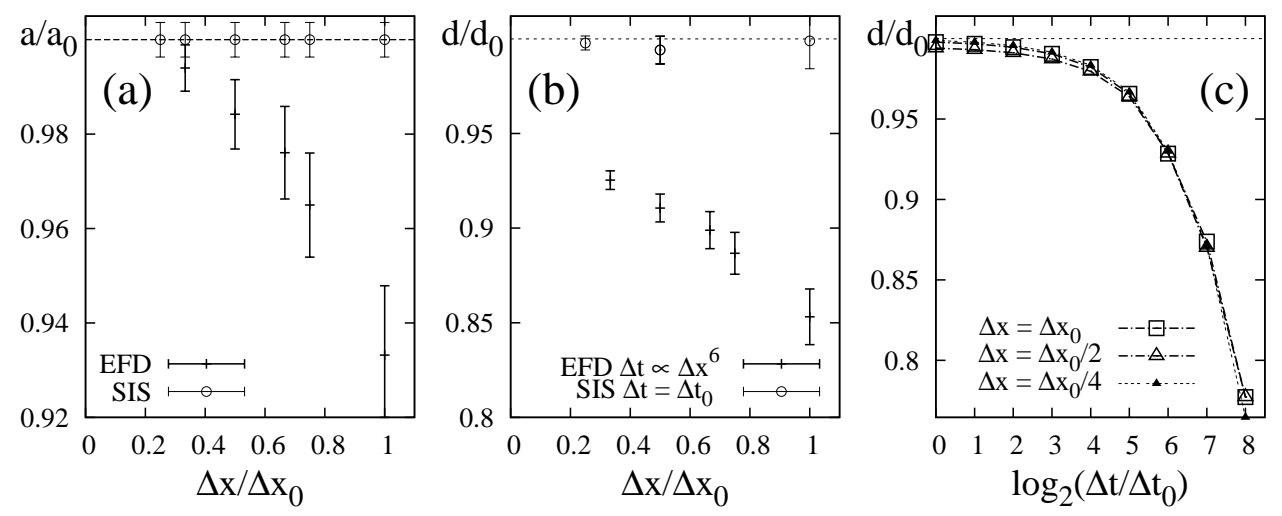

Fig. 3. Effect of the spatial and time resolution on the numerical results obtained with the semi-implicit spectral (SIS) and explicit finite difference (EFD) methods: (a) Normalized interatomic distance $\left(a / a_{0}\right)$ vs. $\Delta x$; (b) normalized diameter $\left(d / d_{0}\right)$ vs. $\Delta x$; (c) normalized diameter $\left(d / d_{0}\right)$ vs. $\Delta t$. Here $a_{0}=7.438 \times(1.0 \pm 0.004)$, and $d_{0}=192.0 \times(1.0 \pm 0.003)$. The error bars are explained in the text. In panel (a), however, for the SIS results we have taken the value and error corresponding to the smallest $\Delta x$, as the SIS solutions for different spatial steps fall virtually on top of each other (see Fig. 4), and thus the solution with the highest spatial resolution can be regarded as the best interpolation scheme.

observed in the investigated $\Delta t$ and $\Delta x$ ranges are as follows:

(i) The interatomic distance $\left[a_{0}=7.438 \times(1.0 \pm 0.004)\right]$ is virtually independent from both the spatial and time steps [Fig. 3(a)];

(ii) The diameter of the crystalline particle at dimensionless time $t=768$ converges to $d_{0}=192.0 \times(1.0 \pm 0.003)$ for $\Delta t \rightarrow 0$, independently of the spatial step [see Figs. 3(b) and (c)].

The virtual independence of the interatomic distance from $\Delta x$ is a direct consequence of the fact that the solutions obtained at different spatial resolutions fall on top of each other with a high accuracy (see Fig. 4). This independence of the SIS solution of spatial steps (in the investigated range) implies convergency for $\Delta x \rightarrow 0$, as might be expected from the exponential convergence of the Fourier-spectral spatial discretization [46]. Indeed, if $\Delta x>\Delta x_{0}$ is used, we see deviations from the closely matching solutions shown in Fig. 4. The particle diameter at fixed time (or the average growth rate) seems to be also independent of the spatial step in the $\Delta x \in[1 / 4,1] \Delta x_{0}$ range. However, it depends on the time step, and converges to a limiting value for $\Delta t \rightarrow 0$ : the difference of diameters obtained with the smallest two time steps is $\sim 0.1 \%$. Remarkably, even for a time step as large as $\Delta t=32 \times \Delta t_{0}$, the average growth rate is only $\sim 3.3 \%$ less than this limiting value. These findings suggest the convergence of the SIS solutions to a limiting solution for $\Delta x \rightarrow 0$ and $\Delta t \rightarrow 0$. We note here that the Fourier-spectral spatial discretization is highly accurate, and in our solutions the numerical error originating from the time stepping 

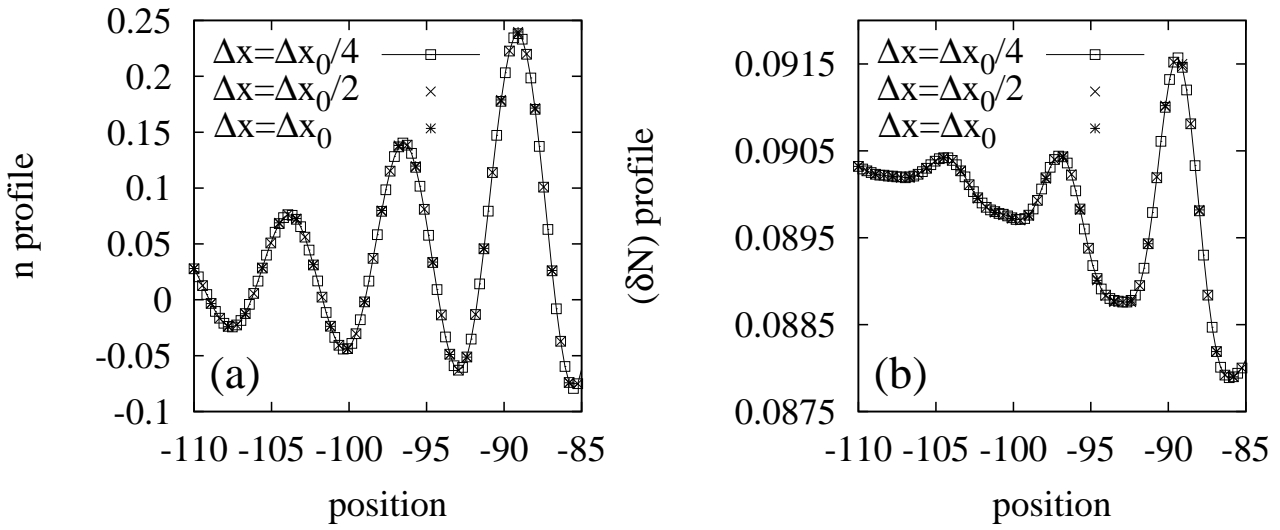

Fig. 4. Cross-sectional profiles $(t=768)$ for the solid-liquid interface in SIS simulations performed using three different mesh spacings and $\Delta t=\Delta t_{0}$ : (a) Total number density $n$, and (b) number density difference $(\delta N)$. Note that the results are fairly independent of the mesh spacing.
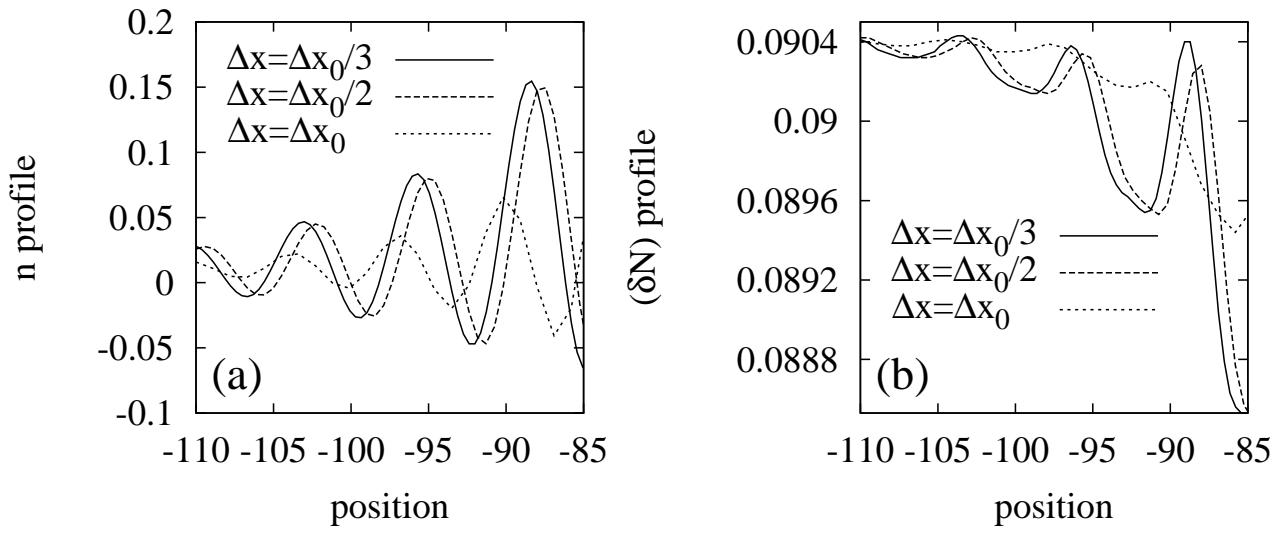

Fig. 5. Cross-sectional profiles $(t=768)$ for the solid-liquid interface in EFD simulations performed using three different mesh spacings and the maximum time steps that ensure numerical stability: (a) Total number density $n$, and (b) number density difference $(\delta N)$. These EFD results refer to similar spatial resolutions and the same region of the crystalline particle as those in Fig. 4. Note that the EFD results depend on the mesh spacing considerably.

seems to dominate. This is hardly surprising considering that the backward Euler time stepping is accurate only to the first order. It is thus expected that the application of time stepping methods that are accurate to higher orders could further improve the accuracy/efficiency of the SIS approach.

Comparison to explicit finite difference method: The total number density $(n)$ and normalized number density difference $(\delta N)$ profiles obtained by the EFD method for three different spatial steps $\left(\Delta x_{0} / 3, \Delta x_{0} / 2\right.$, and $\left.\Delta x_{0}\right)$ are shown in Fig. 5. Remarkably, we see a rather strong dependence on the spatial resolution, though convergence is observed towards the smaller spatial steps. Unfortunately, due to its prohibitively large computation time, we were un- 
able to perform the simulation for $\Delta x_{0} / 4$ and below, even for the very small physical domain chosen for the numerical test. (Note that in these EFD computations the time step had to be varied to ensure numerical stability.) It is remarkable, that a much finer spatial resolution would be needed to ensure the same level of spatial accuracy as provided by Fourier spectral scheme used in the SIS method. However, such EFD computations would only be possible for systems of very small physical size and even then only very short physical times could be covered.

We compare the EFD predictions for the interatomic distance and the diameter to those from the SIS method in Fig. 3. In agreement with the results in Fig. 5, the EFD data for both the $a$ and $d$ vary strongly with resolution. For the interatomic distance, the EFD results approach those from the SIS method from below: The interatomic distances predicted by the two methods seem to converge to values that fall within the range of the combined errors. Remarkably, at $\Delta x=\Delta x_{0}$, the EFD computation underestimates the interatomic distance by $\sim 6 \%$. We wish to note here that the dependence of the EFD results on resolution is likely to be reflected in other physical properties such as the bulk modulus, compressibility, and the free energy realized in the numerical computations. (Note that unlike the conventional phase field methods, where thermodynamics of the bulk phases is an input whos accuracy is usually independent from the accuracy of the applied numerical method, here even the free energy of the bulk phases depend on the accuracy of the numerical scheme applied.) The EFD results for the diameter of the crystalline particle underestimate those from the SIS method by $\sim 7$ to $15 \%$, though they seem to approach the SIS limit $\left(d_{0}\right)$ for decreasing time steps. A linear extrapolation of the EFD data to $\Delta x=0$ yields an $\sim 4 \%$ lower limiting value for the diameter than the corresponding SIS prediction. One cannot, however, rule out that a better convergence would be observed eventually were the spatial step decreased further. We note also that convergence in the empirical test might be limited by the cumulative round-off error, a type of error that becomes especially enhanced for the EFD scheme with decreasing spatial and time steps.

In order to quantify further the differences between the solutions obtained with the same spatial resolution by the two numerical methods, we introduce the scaled $L^{2}$ difference of the Fourier transform of the SIS solution relative to the Fourier transform of the EFD solution, defined as

$$
\sigma_{\chi}=\frac{\sqrt{\left\langle\left(\chi_{S I S}-\chi_{E F D}\right)^{2}\right\rangle}}{\max \left(\chi_{E F D}\right)-\min \left(\chi_{E F D}\right)},
$$

where $\chi=\hat{n}$ or $\widehat{(\delta N)}$, while the quantities with hat denote the Fourier transform of the respective fields. Note that it is advantageous to compute the 
Table 2

Scaled $L^{2}$ difference of the Fourier spectra

\begin{tabular}{rrr}
\hline \hline$\Delta x$ & $\sigma_{\hat{n}}$ & $\sigma_{\widehat{(\delta N)}}$ \\
\hline$\Delta x_{0}$ & $9.8451 \times 10^{-2}$ & $10.9165 \times 10^{-2}$ \\
$3 / 4 \Delta x_{0}$ & $9.2025 \times 10^{-2}$ & $9.8817 \times 10^{-2}$ \\
$2 / 3 \Delta x_{0}$ & $7.6462 \times 10^{-2}$ & $7.6462 \times 10^{-2}$ \\
$1 / 2 \Delta x_{0}$ & $5.5147 \times 10^{-2}$ & $6.1442 \times 10^{-2}$ \\
$1 / 3 \Delta x_{0}$ & $2.3969 \times 10^{-2}$ & $2.8141 \times 10^{-2}$ \\
\hline
\end{tabular}

scaled $L^{2}$ difference for the Fourier transforms, and not for the fields themselves, since both fields are periodic in the solid state, and deviations in the interatomic spacings for the SIS and EFD solutions are also observed. The Fourier tranforms of the solutions have been obtained by 2D FFT. The respective results are presented in Table 2 . We find that the scaled $L^{2}$ difference decreases with decreasing spatial step for both fields $[\hat{n}$ and $\widehat{(\delta N)}]$.

\subsection{Computational efficiency}

Since these calculations are rather costly, it is of interest to compare how efficiently the SIS and EFD methods can be parallelized. (We performed this part of our study on the RISSPO cluster.) First, we determine the effective computational time $\tau$ one needs to compute one time step in a grid point for both methods, which we relate to the full computational time, $t_{\text {comp }}$ needed to solve the equations of motion on an $N \times N$ grid, and for $N_{t}$ time steps as follows: $\tau=t_{\text {comp }} /\left(N^{2} N_{t}\right)$. The results are displayed as a function of the number of the CPU cores in Fig. 6(a). It appears that the computational speed $(1 / \tau)$ is essentially comparable for the SIS and EFD methods, and except for the SIS scheme for small grid sizes on large number of CPU cores, it scales roughly linearly with the number of CPU cores. This means that the computational cost for calculating a time step at a grid point is comparable for SIS and EFD (the EFD cost is smaller by about a factor of $\sim 2.5$ in general).

Next, we compare how fast one can obtain solutions of the same time and spatial resolution using the SIS and EFD methods. For this, we perform computations of the same physical size, up to unit physical time with different spatial resolutions $\left[\Delta x=(1 / 4,1 / 2,1) \cdot \Delta x_{0}\right]$, and at the maximum time step that is stable and accurate enough. The results are compared in Fig. 6(b). While at a modest spatial resolution $\left(\Delta x=\Delta x_{0}\right)$ the computational time for the SIS method is about an order of magnitude smaller than for the EFD scheme (due to the larger time step allowed), for $\Delta x=\Delta x_{0} / 2$ it grows to al- 

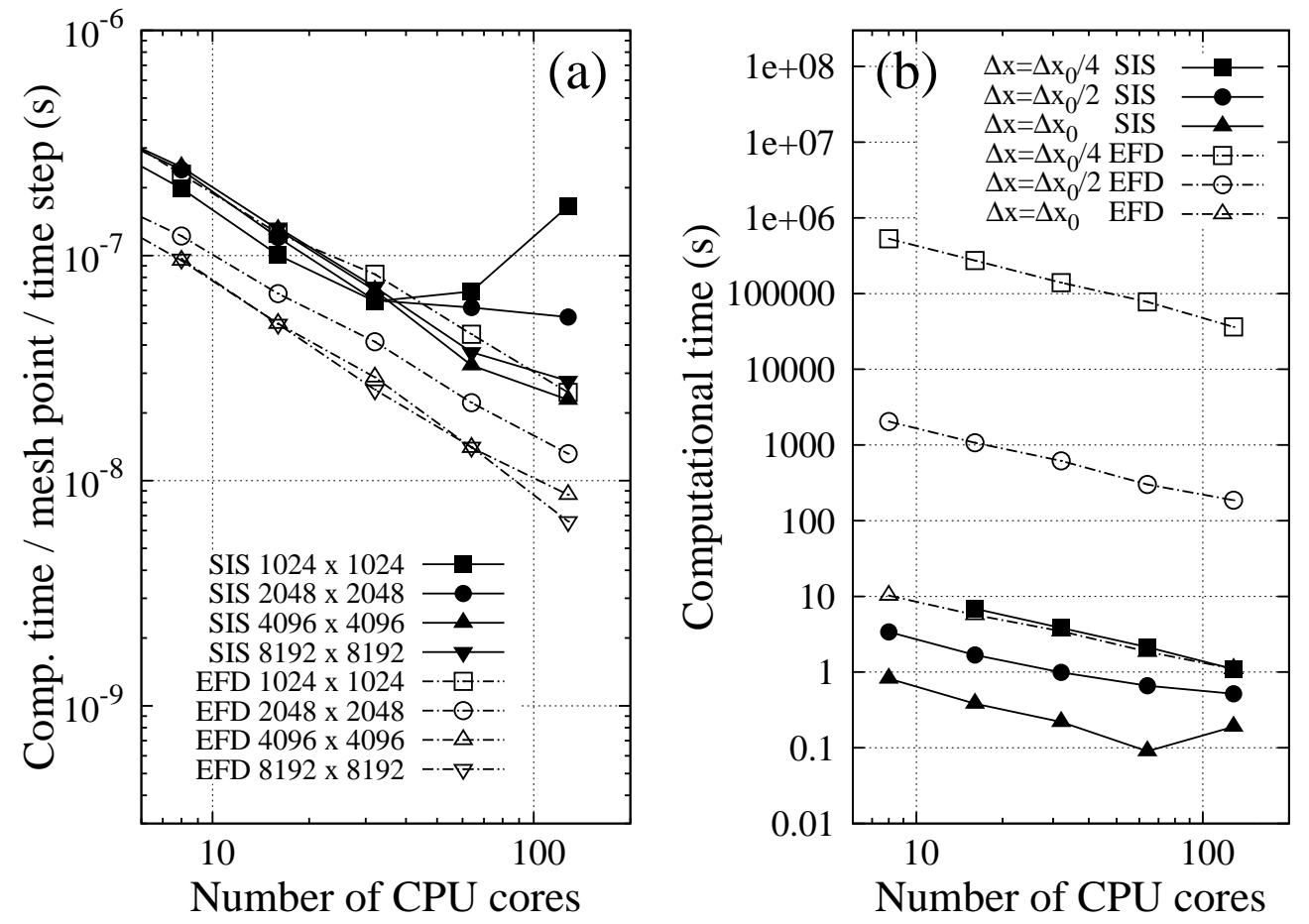

Fig. 6. Scaling properties of the SIS and EFD schemes: (a) Computational cost of a single time step in a single mesh point vs. number of CPU cores; (b) total computation time needed to perform a simulation for a given physical problem (given linear size and physical duration) at different spatial resolutions, as a function of the number of CPU cores.

most 3 orders of magnitude, while for $\Delta x=\Delta x_{0} / 4$ the SIS computation of the same task is nearly 5 orders of magnitude faster. It is worth noting furthermore that, even at the same $\Delta x$ value, the SIS computation provides a considerably more accurate interatomic distance than the EFD scheme (see Fig. 3), suggesting that the SIS method shall be even more preferable, if computations of the same effective numerical error are compared. Considering that the same accuracy of the interatomic distance, which the SIS scheme achieves at $\Delta x_{0}$ cannot be achieved by the EFD metod even at $\Delta x_{0} / 4$, the gain by applying the SIS method is more than 5 orders of magnitude in the computation time. It is also remarkable that except for small grids on a large number of CPU cores, the computation time of SIS scales with the number of the CPU cores as well as for the EFD method $\left(t_{\text {comp }} \propto N_{\text {core }}^{-1}\right)$. For example, in the case of our largest computations (on a 16,384 $\times 16,384$ grid), we have found this type of scaling up to our maximum number of CPU cores, 192, connected with high speed communication. 


\subsection{A couple of practical remarks:}

(a) In the SIS scheme, the addition of colored noise to the equations of motion is fairly straightforward, and does not lead to extra calling of FFT/IFFT.

(b) We find that an accurate solution of the PFC equations is important since the physical properties (e.g., interatomic distance, compressibility, bulk modulus, phase diagram, growth velocity, etc.) appear to depend strongly on computational accuracy. It is in this high accuracy limit required for quantitative calculations, where the proposed SIS method offers the most.

(c) The proposed SIS method is expected to be comparably efficient for equations of motions containing higher oder PDEs that emerge if higher order approximations of the two-particle direct correlation function are incorporated into the PFC model.

\section{Summary}

We have presented an efficient semi-implicit spectral scheme based on a specific operator splitting technique for solving numerically the equations of motion of the binary phase-field crystal model. We have demonstrated the following.

(i) For decreasing time and spatial steps, the solution obtained with the proposed semi-implicit scheme converges to a limiting solution.

(ii) In the range, where computations with the explicit finite difference scheme can be performed, results from the explicit scheme approach those from the semi-implicit spectral scheme with decreasing time and spatial steps.

(iii) Significant acceleration of the computations can be expected if the proposed semi-implicit spectral scheme is used, especially if accurate solutions are needed, in which case the new method can be several orders of magnitude faster than the conventional explicit finite difference scheme.

(iv) Since the proposed method is implicit in the Fourier space, it can be parallelized efficiently: in the investigated size and CPU core number ranges, the computational time scales roughly with the inverse of the number of the CPU cores.

We expect that by applying higher order time stepping, the efficiency of the method can further be improved. Work is underway into this direction. 


\section{Acknowledgments}

This work has been supported by the Hungarian Academy of Sciences under contract OTKA-K-62588 and by the EU FP7 Collaborative Project ENSEMBLE under Contract No. CP-FP-213669-2. T. P. is a grantee of the Bolyai János Scholarship. G. B. acknowledges EPSRC for financial support.

\section{References}

[1] J. D. Gunton, M. San Miguel, and P. S. Sahni, in Phase Transitions and Critical Phenomena, edited by C. Domb and J. L. Lebowitz (Academic Press, New York, 1983), Vol. 8, p. 267.

[2] J. S. Langer, in Solids far from Equilibrium, edited by C. Godrèche (Cambridge University Press, Cambridge, 1992), p. 297.

[3] A. J. Bray, Theory of phase-ordering kinetics, Adv. Phys. 43, 357 (1994).

[4] J. W. Cahn and J. E. Hilliard, Free energy of a nonuniform system. I. Interface free energy, J. Chem. Phys. 28, 258 (1958).

[5] S. M. Allen and J. W. Cahn, A microscopic theory for antiphase boundary motion and its application to antiphase domain coarsening, Acta Metall. 27, 1085 (1979).

[6] W. H. Shih, Z. Q. Wang, X. C. Zeng, and D. Stroud, Ginzburg-Landau theory for the solid-liquid interface of bcc elements, Phys. Rev. A 35, 2611 (1987).

[7] T. M. Rogers, K. R. Elder, and R. C. Desai, Numerical study of the late stages of spinodal decomposition, Phys. Rev. B 37, 9638 (1988).

[8] Y. Oono and S. Puri, Study of phase-separation dynamics by use of cell dynamical systems. I. Modeling, Phys. Rev. A 38, 434 (1998).

[9] K.-A. Wu, A. Karma, J. J. Hoyt, and M. Asta, Ginzburg-Landau theory of crystalline anisotropy for bcc-liquid interfaces, Phys. Rev. B 73, 094101 (2006).

[10] K. R. Elder, M. Katakowski, M. Haataja, and M. Grant, Modeling elasticity in crystal growth, Phys. Rev. Lett. 88, 245701 (2002).

[11] K. R. Elder and M. Grant, Modeling elastic and plastic deformations in nonequilibrium processing using phase field crystals, Phys. Rev. E 70, 051605 (2004).

[12] For review on classical DFT of crystallization see: D. W. Oxtoby, Crystallization of liquids: a density functional approach, in Liquids, Freezing, and Glass Transition, edited by J. P. Hansen, D. Levesque and J. Zinn-Justin (North Holland, Amsterdam, 1991), Vol. I, p. 145. 
[13] J. Swift and P. C. Hohenberg, Hydrodynamic fluctuations at the convective instability, Phys. Rev. A 15, 319 (1977).

[14] S. A. Brazovsky, Phase transition of an isotropic system to an inhomogeneous state, Zh. Eksp. Teor. Fiz. 68, 175 (1975).

[15] N. Provatas, J. A. Dantzig, B. Athreya, P. Chan, P. Stefanovic, N. Goldenfeld, and K. R. Elder, Using the phase-feild crystal method in the multi-scale modeling of microstructure evolution, JOM 59, 83 (2007).

[16] K. R. Elder, N. Provatas, J. Berry, P. Stefanovic, and M. Grant, Phase-field crystal modeling and classical density functional theory of freezing, Phys. Rev. B. 75, 064107 (2007).

[17] M. Cheng and J. A. Warren, An efficient algorithm for solving the phase field crystal method, J. Comput. Phys. 227, 6241 (2008).

[18] N. Goldenfeld, B. P. Athreya, and J. A. Dantzig, Renormalization group approach to multiscale simulation of polycrystalline materials using the phase field crystal model, Phys. Rev. E 72, 020601 (2005).

[19] N. Provatas, M. Greenwood, B. Athreya, and N. Goldenfeld, Multiscale Modeling of Solidification: Phase-Field Methods to Adaptive Mesh Refinement, Int. J. Mod. Phys. B 59, 4525 (2005).

[20] B. P. Athreya, N. Goldenfeld, J. A. Dantzig, M. Greenwood, and N. Provatas, Adaptive mesh computation of polycrystalline pattern formation using a renormalization-group reduction of the phase-field crystal model, Phys. Rev. E 76, 056706 (2007).

[21] G. Strang, On the construction and comparison of different splitting schemes. SIAM J. Numer. Anal. 5, 506 (1968).

[22] G. I. Marchuk, Methods of Splitting (Nauka, Moscow, 1988).

[23] C. I. Christov and R. S. Marinova, Implicit vectorial operator splitting for incompressible Navier - Stokes equations in primitive variables. J. Comput. Technologies 6, 92 (2001).

[24] M. Mimura, T. Nakaki, and K. Tomoeda, A numerical approach to interface curves for some nonlinear diffusion equations. Jpn. J. Appl. Math. 1, 93 (1984).

[25] E. R. Jakobsen, K. H. Karlsen, and N. H. Risebro, On the convergence rate of operator splitting for Hamilton - Jacobi equations with source terms. SIAM J. Numer. Anal. 39, 499 (2001).

[26] K. H. Karlsen, and N. H. Risebro, Unconditionally stable methods for Hamilton - Jacobi equations. J. Comput. Phys. 180, 710 (2002).

[27] K. H. Karlsen, K.-A. Lie, J. R. Natvig, H. F. Nordhaug, and H. K. Dahle, Operator splitting methods for systems of convection- diffusion equations: nonlinear error mechanisms and correction strategies. J. Comput. Phys. 173, 636 (2001). 
[28] R. S. Marinova, C. I. Christov, and T. T. Marinov, A fully coupled solver for incompressible Navier - Stokes equations using operator splitting. Int. J. Comput. Fluid Dynamics 17, 371 (2003).

[29] I. Faragó and Á. Havasi, On the convergence and local splitting error of different splitting schemes. Prog. Comput. Fluid Dynamics 5, 495 (2005).

[30] P. Csomós, and I. Faragó, Error analysis of the numerical solution of split differential equations, Math. Comput. Model. 48, 1090 (2008).

[31] J. Woodhead-Galloway and T. Gaskel, Structure of mixtures of simple fluids, J. Phys. C 1, 1472 (1968)

[32] D. Musicki, On canonical formalism in field theory with derivatives of higher order-canonical transformations Physica A 11, 39 (1978).

[33] W. Hundsdorfer, J. G. Verwer, Numerical Solution of Time-Dependent Advection-Diffusion-Reaction Equations (Springer-Verlag, Berlin, 2003).

[34] I. Faragó, Splitting methods and their application to the abstract Cauchy problems, in Lecture Notes in Comput. Science, (Springer-Verlag, Berlin, 2005), Vol. 3401, p. 35.

[35] P. Csomós, I. Faragó, and Á. Havasi, Weighted sequential splittings and their analysis, in Proceedings of NMCM-2002, Comput. Math. Appl. 50, 1017 (2005).

[36] J. G. Levin, M. Iskandarani, and D. B. Haidvogel, A spectral filtering procedure for eddy-resolving simulations with a spectral element ocean model, J. Comput. Phys. 137, 130 (1997).

[37] J. Garcia-Ojalvo, J. M. Sancho, and L. Remirez-Piscina, Generation of spatiotemporal colored noise, Phys. Rev. A 46, 4670 (1992).

[38] J. M. Sancho, J. Garcia-Ojalvo, and H. Guo, Non-equilibrium Ginzburg-Landau model driven by colored noise, Physica D 113, 331 (1998).

[39] M. Frigo and S. G. Johnson, The design and implementation of FFTW3, Proc. of the IEEE 93, 216 (2005).

[40] M. D. Abramoff, P. J. Magelhaes, and S. J. Ram, Image Processing with ImageJ, Biophotonics Int. 11, 36 (2004).

[41] C. Budd, O. Koch, and E. Weinmüller, Computation of self-similar solution profiles for the nonlinear Schrödinger equation. Computing 77, 335 (2006).

[42] R. L. Davidchack and B. B. Laird, Simulation of the hard-sphere crystal-melt interface, J. Chem. Phys. 108, 9452 (1998).

[43] H. Ramalingam, M. Asta, A. van de Walle, and J. J. Hoyt, Atomic-scale study of equilibrium solute adsorption at alloy solid-liquid interfaces, Interface Sci. 10, 149 (2002).

[44] D. Y. Sun, M. Asta, and J. J. Hoyt, Crystal-melt free energies and mobilitites in fcc and bcc Fe, Phys. Rev. B 69, 174103 (2004). 
[45] J. R. Morris, Complete mapping of the anisotropy of the crystal-melt interface in Al, Phys. Rev. B 66, 144104 (2002).

[46] D. Gottlieb and S. A. Orszag, Numerical Analysis of Spectral Methods: Theory and Application (SIAM-CBMS, Philadelphia, PA, 1977). 